\title{
Analysis of a Computer System with Software Redundancy Subject to Hardware Preventive Maintenance
}

\author{
V. J. Munday* \\ *Department of Statistics, \\ Ramjas College, University of Delhi, \\ Delhi - 110007, India
}

\author{
Permila $^{\#}$ \\ \#Department of Statistics, \\ Govt. College for Women, \\ Rohtak - 124001, India
}

\author{
S.C. Malik ${ }^{\$}$ \\ ${ }^{\$}$ Department of Statistics, \\ M.D. University, \\ Rohtak - 124001, (India)
}

\begin{abstract}
The present work is devoted to the analysis of a computer system with software redundancy subject to hardware preventive maintenance. For this purpose, a system model has been developed by considering software redundancy. There is an independent hardware and software failure in the system model. The preventive maintenance of the hardware component has been conducted after a specific operation time ' $t$ ' (called maximum operation time). A single server is provided immediately to the system for carrying out hardware repair, software up-gradation and hardware preventive maintenance as and when needed. The repair activities conducted by the server are perfect. The failure time of the hardware and software components follow negative exponential distribution whereas the distributions for hardware repair, software up-gradation and preventive maintenance times are taken as arbitrary with different probability density functions. Some important reliability characteristics of the system models have been examined stochastically in steady state for particular values of various parameters and costs by using semi-Markov process and regenerative point technique. Graphs are drawn to depict the behaviour of mean time to system failure (MTSF), availability and profit function under different sets of assumptions on the parameters. The profit of the present model has also been compared with the model as discussed in the research paper of [5].
\end{abstract}

Keywords - Computer System; Software Redundancy; Upgradation; Preventive Maintenance, Profit Analysis and Stochastic Modelling

\section{INTRODUCTION}

In past few decades, the importance of computer widely increases in most of areas such as companies, medicals, banks, etc. In the previous research based on computer system, some stochastic models for a computer system have been developed and analyzed in steady state by considering the aspects of component wise redundancy in cold standby, priority in repair disciplines and maximum repair time to hardware. And, a computer system would be more profitable if redundancy is provided to the hardware along with maximum repair time rather than redundancy to the software. [1] examined a two-unit standby redundant system with preventive maintenance. [2] Analyzed a two dissimilar cold standby system with preventive maintenance and replacement of standby. On the other hand, the deterioration rate of a system can be reduced by conducting its preventive maintenance after a maximum operation time. [3] obtained reliability measures of a system under preventive maintenance. Hence, it becomes necessary to examine the effect of preventive maintenance on reliability measures of a computer system with the concepts of preventive maintenance and software redundancy. However, [4] tried to develop stochastic models of operating systems with preventive maintenance and priority subject to maximum operation and repair times. [6] established reliability measures of a computer system with hardware redundancy subject to preventive maintenance. The present work is devoted to the analysis of a computer system with software redundancy subject to hardware preventive maintenance. For this purpose, a system model has been developed by considering software redundancy in cold standby with the concept of hardware preventive maintenance. Hardware and software failures are independent in the system model. The preventive maintenance of the hardware component has been conducted after a specific operation time ' $t$ ' (called maximum operation time). There is a single server who visits the system immediately for carrying out hardware repair, software up-gradation and hardware preventive maintenance as and when needed. The repair activities conducted by the server are perfect. The failure time of the hardware and software components follow negative exponential distribution whereas the distributions for hardware repair, software up-gradation and preventive maintenance times are taken as arbitrary with different probability density functions. Some important reliability characteristics of the system models have been examined 
stochastically in steady state for particular values of various parameters and costs by using semi-Markov process and regenerative point technique. Graphs are drawn to depict the behaviour of mean time to system failure (MTSF), availability and profit function under different sets of assumptions on the parameters. The profit of the present model has also been compared with the model as discussed in the research paper of [5].

\section{SOME IMPORTANT NOTATIONS}

$\mathrm{O}:$ : Computer system is operative

Scs : Software is in cold standby

$\mathrm{a} / \mathrm{b} \quad$ : Probability that the system has hardware / software failure

$\lambda_{1} / \lambda_{2}: \quad$ : Hardware/Software failure rate

$\beta_{0} \quad:$ The rate by which hardware component undergoes for preventive maintenance

HFUr /HFWr : The hardware is failed and under/waiting for repair

SFUg/SFWUg : The software is failed and under/waiting for up-gradation

HFUPm /HFWPm : The hardware is under/waiting for preventive maintenance

HFUR/HFWR : The hardware is failed and continuously under/ waiting for repair from previous state

SFUG/SFWUG : The software is failed and continuously under /waiting for up- gradation from previous state

HFUPM/HFWPM : The hardware is under/ waiting for preventive maintenance continuously from previous state

$\mathrm{g}(\mathrm{t}) / \mathrm{G}(\mathrm{t}) \quad$ : pdf/cdf of hardware repair time

$\mathrm{f}(\mathrm{t}) / \mathrm{F}(\mathrm{t}) \quad:$ pdf/cdf of software up-gradation time

$\mathrm{m}(\mathrm{t}) / \mathrm{M}(\mathrm{t}) \quad$ : pdf/cdf of hardware preventive maintenance time

$\mathrm{q}_{\mathrm{ij}}(\mathrm{t}) / \mathrm{Q}_{\mathrm{ij}}(\mathrm{t})$ : pdf/cdf of first passage time from regenerative state $S_{i}$ to a regenerative state $S_{j}$ or to a failed state $S_{j}$ without visiting any other regenerative state in $(0, \mathrm{t}]$

$\mathrm{q}_{\mathrm{ij} . \mathrm{k}}(\mathrm{t}) / \mathrm{Q}_{\mathrm{ij} . \mathrm{k}}(\mathrm{t})$ : pdf/cdf of direct transition time from regenerative state $S_{i}$ to a regenerative state $S_{j}$ or to a failed state $S_{j}$ visiting state $S_{k}$ once in $(0, t]$

$M_{i}(t)$ : Probability that the system up initially in state $S_{i} \in E$ is up at time $t$ without visiting to any regenerative state

$\mathrm{W}_{\mathrm{i}}(\mathrm{t})$ : Probability that the server is busy in the state $S_{i}$ up to time ' $t$ ' without making any transition to any other regenerative state or returning to the same state via one or more non-regenerative states.

$\mu_{\mathrm{i}}$ : The mean sojourn time in state $S_{i}$ which is given by

$$
\mu_{i}=E(T)=\int_{0}^{\infty} P(T>t) d t=\sum_{j} m_{i j},
$$

where $T$ denotes the time to system failure.

$\mathrm{m}_{\mathrm{ij}}$ : Contribution to mean sojourn time $\left(\mu_{\mathrm{i}}\right)$ in state $\mathrm{S}_{\mathrm{i}}$ when system transits directly to state $\mathrm{S}_{\mathrm{j}}$ so that

$$
\mu_{i}=\sum_{j} m_{i j} \text { and } m_{i j}=\int_{0}^{\infty} t d Q_{i j}(t)=-q_{i j}^{*^{\prime}}(0)
$$

$\& /$ C) : Symbol for Laplace - Stieltjes convolution

\section{/ Laplace convolution}

*/** : Symbol for Laplace Transformation (LT) /Laplace Stieltjes Transformation (LST)

$\phi_{i}(t)$ : cdf of first passage time from regenerative state $S_{i}$ to a failed state. Regarding the failed state as absorbing state

$A_{i}(t)$ : Probability that the system is in up-state at instant ' $t$ ' given that the system entered regenerative state $S_{i}$ at $t=0$.

$B_{i}^{H}(t)$ : Probability that the server is busy in repairing the unit due to hardware failure at an instant ' $t$ ' given that the system entered state $S_{i}$ at $t=0$.

$B_{i}^{S}(\mathrm{t})$ : Probability that the server is busy in upgradation of the software at an instant ' $t$ ' given that the system entered the regenerative state $S_{i}$ at $t=0$.

$B_{i}^{P m}(t)$ : Probability that the server is busy in preventive maintenance of the hardware given that the system entered state $S_{i}$ at $\mathrm{t}=0$.

$N H R_{i}(t)$ : Expected number of hardware repairs by the server in $(0, t]$ given that the system entered the regenerative state $S_{i}$ at $t=0$.

$\mathrm{NSU}_{\mathrm{i}}(\mathrm{t})$ : Expected number of software upgradations in $(0, \mathrm{t}]$ given that the system entered the regenerative state $S_{i}$ at $t=0$.

$\mathrm{NHPm}_{i}(t)$ :Expected number of hardware preventive maintenances by the server in $(0, \mathrm{t}]$ given that the system entered the regenerative state $S_{i}$ at $t=0$.

$\mathrm{K}_{0}$ : Revenue per unit up - time of the system

$\mathrm{K}_{1}$ : Cost per unit time for which server is busy due hardware repair

$\mathrm{K}_{2}$ : Cost per unit time for which server is busy due software Up-gradation

$\mathrm{K}_{3}$ : Cost per unit repair of the failed hardware

$\mathrm{K}_{4}$ : Cost per unit up-gradation of the failed software

$\mathrm{K}_{5}$ : Cost per unit time for which server is busy due hardware preventive maintenance

$\mathrm{K}_{6}$ : Cost per unit preventive maintenance of the failed hardware

$\mathrm{P}$ : Profit of the present model

P1 : Profit of the system model [5] 


\section{ANALYSIS OF SYSTEM MODEL}

The state transition diagram is shown in the following figure:

State Transition Diagram

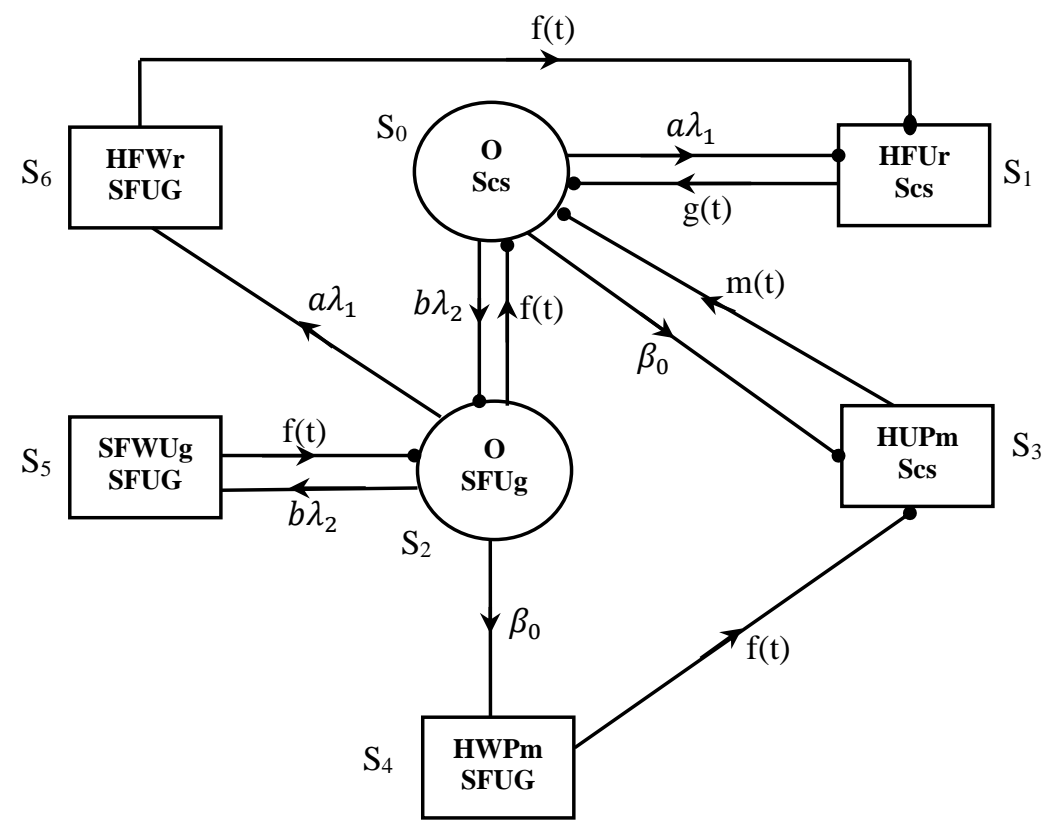

Up-State

Failed State

- Regenerative Point

Fig. 1

\section{A. Transition Probabilities and Mean Sojourn Times}

Simple probabilistic considerations yield the following expressions for the non-zero elements.

$p_{i j}=Q_{i j}(\infty)=\int_{0}^{\infty} q_{i j}(t) d t$

$p_{01}=\frac{a \lambda_{1}}{a \lambda_{1}+b \lambda_{2}+\beta_{0}}, \quad p_{02}=\frac{b \lambda_{2}}{a \lambda_{1}+b \lambda_{2}+\beta_{0}}, \quad p_{03}=$ $\frac{\beta_{0}}{a \lambda_{1}+b \lambda_{2}+\beta_{0}}$

$p_{10}=g^{*}(0), \quad p_{20}=f^{*}\left(a \lambda_{1}+b \lambda_{2}+\beta_{0}\right), \quad p_{24}=$ $\frac{\beta_{0}}{a \lambda_{1}+b \lambda_{2}+\beta_{0}}\left\{1-f^{*}\left(a \lambda_{1}+b \lambda_{2}+\beta_{0}\right)\right\}$,

$p_{25}=\frac{b \lambda_{2}}{a \lambda_{1}+b \lambda_{2}+\beta_{0}}\left\{1-f^{*}\left(a \lambda_{1}+b \lambda_{2}+\beta_{0}\right)\right\}, \quad p_{26}=$ $\frac{a \lambda_{1}}{a \lambda_{1}+b \lambda_{2}+\beta_{0}}\left\{1-f^{*}\left(a \lambda_{1}+b \lambda_{2}+\beta_{0}\right)\right\}, p_{30}=$ $m^{*}(0), p_{43}=p_{52}=f^{*}(0)$

For $\mathrm{f}(\mathrm{t})=\theta e^{-\theta t}, \mathrm{~g}(\mathrm{t})=\alpha e^{-\alpha t}$ and $\mathrm{m}(\mathrm{t})=\gamma e^{-\gamma t}$

we have

$p_{23.4}=\frac{\beta_{0}}{a \lambda_{1}+b \lambda_{2}+\beta_{0}}\left\{1-f^{*}\left(a \lambda_{1}+b \lambda_{2}+\beta_{0}\right)\right\} f(t)$

$p_{22.5}=\frac{b \lambda_{2}}{a \lambda_{1}+b \lambda_{2}+\beta_{0}}\left\{1-f^{*}\left(a \lambda_{1}+b \lambda_{2}+\beta_{0}\right)\right\} f(t)$

$p_{21.6}=\frac{a \lambda_{1}}{a \lambda_{1}+b \lambda_{2}+\beta_{0}}\left\{1-f^{*}\left(a \lambda_{1}+b \lambda_{2}+\beta_{0}\right)\right\} f(t)$

But, $\mathrm{f}^{*}(0)=\mathrm{g}^{*}(0)=\mathrm{m}^{*}(0)=1$ and $\mathrm{a}+\mathrm{b}=1$

It can be easily verified that

$\mathrm{p}_{01}+\mathrm{p}_{02}+\mathrm{p}_{03}=\mathrm{p}_{10}=\mathrm{p}_{20}+\mathrm{p}_{24}+\mathrm{p}_{25}+\mathrm{p}_{26}=\mathrm{p}_{20}+\mathrm{p}_{23.4}+\mathrm{p}_{22.5}+\mathrm{p}_{21.6}=$ $\mathrm{p}_{30}=\mathrm{p}_{43}=\mathrm{p}_{52}=1$
The mean sojourn times $\left(\mu_{\mathrm{i}}\right)$ in the state $S_{\mathrm{i}}$ are

$\mu_{0}=\frac{1}{a \lambda_{1}+b \lambda_{2}+\beta_{0}} \quad \mu_{1}=\frac{1}{\alpha} \quad \mu_{2}=\frac{1}{a \lambda_{1}+b \lambda_{2}+\beta_{0}+\theta}$ $\mu_{3}=\frac{1}{\gamma}$

Also

$\mu_{0}=m_{01}+m_{02}+m_{03}, \quad \mu_{1}=m_{10}$,

$\mu_{2}=m_{20}+m_{24}+m_{25}+m_{26}$

And $\mu_{2}^{\prime}=m_{20}+m_{21.6}+m_{22.5}+m_{23.4}$

B. Reliability and Mean Time to System Failure (MTSF)

The recursive relations for $\phi_{i}(t)$ are as follows:

$\phi_{0}(\mathrm{t})=\mathrm{Q}_{01}(\mathrm{t})+\mathrm{Q}_{02}(\mathrm{t}) \& \phi_{2}(\mathrm{t})+\mathrm{Q}_{03}(\mathrm{t})$

$\phi_{2}(\mathrm{t})=\mathrm{Q}_{20}(\mathrm{t}) \& \phi_{0}(\mathrm{t})+\mathrm{Q}_{24}(\mathrm{t})+\mathrm{Q}_{25}(\mathrm{t})+\mathrm{Q}_{26}(\mathrm{t})(4)$

Taking LST of above relations (4) and solving for $\phi_{0}^{* *}(s)$, we have

$R^{*}(s)=\frac{1-\phi_{0}^{* *}(s)}{s}$

The reliability of the system model can be obtained by taking Laplace inverse transform of the above equation. The mean time to system failure (MTSF) is given by

$M T S F=\lim _{s \rightarrow 0} \frac{1-\phi_{0}^{* *}(s)}{s}=\frac{N_{1}}{D_{1}}$

Where $N_{1}=\mu_{0}+p_{02}$ and $D_{1}=1-p_{02} p_{20}$

(6) 
C. Steady State Availability

The recursive relations for $A_{i}(t)$ are given as:

$\mathrm{A}_{0}(\mathrm{t})=\mathrm{M}_{0}(\mathrm{t})+\mathrm{q}_{01}(\mathrm{t})\left(\mathrm{A}_{1}(\mathrm{t})+\mathrm{q}_{02}(\mathrm{t})\left(\mathrm{C} \mathrm{A}_{2}(\mathrm{t})+\mathrm{q}_{03}(\mathrm{t})(\mathrm{C}\right.\right.$ $\mathrm{A}_{3}(\mathrm{t})$

$\mathrm{A}_{1}(\mathrm{t})=\mathrm{q}_{10}(\mathrm{t}) \subset \mathrm{A}_{0}(\mathrm{t})$

$\mathrm{A}_{2}(\mathrm{t})=\mathrm{M}_{2}(\mathrm{t})+\mathrm{q}_{20}(\mathrm{t})\left(\mathrm{C} \mathrm{A}_{0}(\mathrm{t})+\mathrm{q}_{21.6}(\mathrm{t})\left(\mathrm{C} \mathrm{A}_{1}(\mathrm{t})+\mathrm{q}_{22.5}(\mathrm{t})(C)\right.\right.$

$\mathrm{A}_{2}(\mathrm{t})+\mathrm{q}_{23.4}(\mathrm{t})\left(\mathrm{C} \mathrm{A}_{3}(\mathrm{t})\right.$

$\mathrm{A}_{3}(\mathrm{t})=\mathrm{q}_{30}(\mathrm{t}) \odot \mathrm{A}_{0}(\mathrm{t})$

where

$\mathrm{M}_{0}(\mathrm{t})=e^{-\left(a \lambda_{1}+b \lambda_{2}+\beta_{0}\right) t}$

$\mathrm{M}_{2}(\mathrm{t})=e^{\left(a \lambda_{1}+b \lambda_{2}+\beta_{0}\right) t} \frac{,}{F(t)}$

Taking LT of equation (7) and solving for $A_{0}^{*}(s)$, the steady state availability is given by $A_{0}(\infty)=\lim _{s \rightarrow 0} s A_{0}^{*}(s)$ $=\frac{N_{2}}{D_{2}}$

Where

$N_{2}=\mu_{0}\left(1-\mathrm{p}_{22.5}\right)+\mathrm{p}_{02} \mu_{2}$

$D_{2}=\mu_{0}\left(1-p_{22.5}\right)+\mu_{1}\left(p_{01}\left(1-p_{22.5}\right)+\right.$

$\left.p_{02} p_{21.6}\right)+p_{02} \mu_{2}^{\prime}+\mu_{3}\left(p_{03}\left(1-p_{22.5}\right)+p_{23.4}\right)$

\section{Busy Period of the Server}

(a). Due to Hardware Repair

The recursive relations for $B_{i}^{H}(t)$ are as follows:

$B_{0}^{H}(t)=q_{01}(t) \subseteq B_{1}^{H}(t)+q_{02}(t) \mathbb{C} B_{2}^{H}(t)$

$$
+q_{03}(t) \subseteq B_{3}^{H}(t)
$$

$B_{1}^{H}(t)=W_{1}^{H}(t)+q_{10}(t) \subseteq B_{0}^{H}(t)$

$B_{2}^{H}(t)=q_{20}(t) \subset B_{0}^{H}(t)+q_{21.6}(t) \Subset B_{1}^{H}(t)+$

$q_{22.5}(t) \subset B_{2}^{H}(t)+q_{23.4}(t) \subset B_{3}^{H}(t)$

$B_{3}^{H}(t)=q_{30}(t) \mathbb{C} B_{0}^{H}(t)$

where $W_{1}^{H}(t)=\overline{G(t)}$

\section{(b). Due to Software Up-gradation}

The recursive relations for $B_{i}^{S}(t)$ are as follows:

$B_{0}^{S}(t)=q_{01}(t) \subseteq B_{1}^{S}(t)+q_{02}(t) \subseteq B_{2}^{S}(t)+q_{03}(t) \subseteq B_{3}^{S}(t)$

$B_{1}^{S}(t)=q_{10}(t) \subset B_{0}^{S}(t)$

$B_{2}^{S}(t)=W_{2}^{S}(t)+q_{20}(t) \subset B_{0}^{S}(t)+q_{21.6}(t) \subset B_{1}^{S}(t)+$

$q_{22.5}(t) \subseteq B_{2}^{S}(t)+q_{23.4}(t) \oplus B_{3}^{S}(t)$

$B_{3}^{S}(t)=q_{30}(t) \mathbb{C} B_{0}^{S}(t)$

where $W_{2}^{S}(t)=e^{\left(a \lambda_{1}+b \lambda_{2}+\beta_{0}\right) t} \overline{F(t)}+$

$\left(a \lambda_{1} e^{\left(a \lambda_{1}+b \lambda_{2}+\beta_{0}\right) t}(\mathrm{C}) \overline{F(t)}+\right.$

$\left(b \lambda_{2} e^{\left(a \lambda_{1}+b \lambda_{2}+\beta_{0}\right) t}(\mathcal{C}) \overline{F(t)}+\left(\beta_{0} e^{\left(a \lambda_{1}+b \lambda_{2}+\beta_{0}\right) t}(1) \overline{F(t)}\right.\right.$

\section{(c). Due to Preventative Maintenance}

The recursive relations for $B_{i}^{P m}(t)$ are as follows:

$B_{0}^{P m}(t)=q_{01}(t) \subset B_{1}^{P m}(t)$

$$
+q_{02}(t) \subset B_{2}^{P m}(t)+q_{03}(t) \subset B_{2}^{P m}(t)
$$

$B_{1}^{P m}(t)=q_{10}(t) \subseteq B_{0}^{P m}(t)$

$B_{2}^{P m}(t)=q_{20}(t) \subseteq B_{0}^{P m}(t)+q_{21.6}(t) \subseteq B_{1}^{P m}(t)+$

$$
q_{22.5}(t) \odot B_{1}^{P m}(t)+q_{23.4}(t) \subseteq B_{1}^{P m}(t)
$$

$B_{3}^{P m}(t)=W_{3}^{P m}(t)+q_{30}(t) \Subset B_{0}^{P m}(t)$

where $W_{3}^{R p}(t)=\overline{M(t)}$

Taking LT of relations (10), (11) \& (12), solving

for $B_{0}^{H^{*}}(t), B_{0}^{S^{*}}(t)$ and $B_{0}^{P m^{*}}(t)$. The time for which server is busy due to repairs, up-gradations and preventative maintenances respectively are given by

$$
\begin{aligned}
& B_{0}^{H}(t)=\lim _{s \rightarrow 0} s B_{0}^{H^{*}}(t)=\frac{N_{3}^{H}}{D_{2}} \\
& B_{0}^{S}(t)=\lim _{s \rightarrow 0} s B_{0}^{S^{*}}(t)=\frac{N_{3}^{S}}{D_{2}} \\
& B_{0}^{P m}(t)=\lim _{s \rightarrow 0} s B_{0}^{P m^{*}}(t)=\frac{N_{3}^{P m}}{D_{2}}
\end{aligned}
$$

where $N_{3}^{H}=p_{01}\left(p_{10}\left(1-p_{22.5}\right)+p_{02} p_{21.6}\right) W_{1}^{H^{*}}(0)$ $N_{3}^{S}=p_{02} W_{2}^{S^{*}}$

$$
N_{3}^{P m}=\left(p_{02} p_{23.4} p_{30}+p_{03} p_{30}\left(1-p_{22.5}\right)\right) W_{3}^{P m^{*}}(0)
$$

and $D_{2}$ is already mentioned.

\section{E. Expected Number of Hardware Repairs}

The recursive relations for $N H R_{i}(t)$ are given as: $N H R_{0}(t)=Q_{01}(t) \& N H R_{1}(t)+Q_{02}(t) \& N H R_{2}(t)+$ $Q_{03}(t) \& \mathrm{NHR}_{3}(t)$

$$
\begin{aligned}
N H R_{1}(t)= & Q_{10}(t) \&\left(1+N H R_{0}(t)\right) \\
N H R_{2}(t)= & Q_{20}(t) \& N H R_{0}(t)+Q_{21.6}(t) \& N H R_{1}(t) \\
& \quad+Q_{22.5}(t) \& N H R_{2}(t) \\
& +Q_{23.4}(t) \& N H R_{3}(t)
\end{aligned}
$$

Taking LST of relations (17) and solving for $N H R_{0}^{* *}(s)$. The expected number of hardware repairs are given by

$$
N H R_{0}=\lim _{s \rightarrow 0} s N H R_{0}^{* *}(s)=\frac{N_{4}}{D_{2}}
$$

where $\quad N_{4}=p_{01}\left(p_{10}\left(1-p_{22.5}\right)+\right.$ $\left.p_{02} p_{21.6}\right)$ and $D_{2}$ is already mentioned.

\section{F. Expected Number of Software Up-gradations}

The recursive relations for $\mathrm{NSU}_{\mathrm{i}}(\mathrm{t})$ are given as: $\mathrm{NSU}_{0}(\mathrm{t})=\mathrm{Q}_{01}(\mathrm{t}) \& \mathrm{NSU}_{1}(\mathrm{t})+\mathrm{Q}_{02}(\mathrm{t}) \& \mathrm{NSU}_{2}(\mathrm{t})+$

$\mathrm{Q}_{03}(\mathrm{t}) \& \mathrm{NSU}_{3}(\mathrm{t})$

$$
\begin{aligned}
& \mathrm{NSU}_{1}(\mathrm{t})=\mathrm{Q}_{10}(\mathrm{t}) \& \mathrm{NSU}_{0}(\mathrm{t}) \\
& \mathrm{NSU}_{2}(\mathrm{t})=\mathrm{Q}_{20}(\mathrm{t}) \&\left(1+\mathrm{NSU}_{0}(\mathrm{t})\right)+\mathrm{Q}_{21.6}(\mathrm{t}) \& \\
& \left(1+\mathrm{NSU}_{1}(\mathrm{t})\right)+\mathrm{Q}_{22.5}(\mathrm{t}) \&\left(1+\mathrm{NSU}_{2}(\mathrm{t})\right)+\mathrm{Q}_{23.4}(\mathrm{t}) \& \\
& \left(1+\mathrm{NSU}_{3}(\mathrm{t})\right) \\
& \mathrm{NSU}_{3}(\mathrm{t})=\mathrm{Q}_{30}(\mathrm{t}) \& \mathrm{NSU}_{0}(\mathrm{t})
\end{aligned}
$$

LST of relations (20) and solving for $\operatorname{NSU}_{0}^{* *}(\mathrm{~s})$. The expected numbers of software up-gradations are given by

$\mathrm{NSU}_{0}(\infty)=\lim _{\mathrm{s} \rightarrow 0} \mathrm{sNSU}_{0}^{* *}(\mathrm{~s})=\frac{\mathrm{N}_{5}}{\mathrm{D}_{2}}$

Where $\mathrm{N}_{5}=\mathrm{p}_{02}$ and $\mathrm{D}_{2}$ is already mentioned. (22)

G. Expected Number of Preventative Maintenance The recursive relations for $\mathrm{NHPm}_{i}(t)$ are given as: $N H P m_{0}(t)=Q_{01}(t) \& N_{H P m}(t)+Q_{02}(t) \&$ $\mathrm{NHPm}_{2}(t)+Q_{03}(t) \& \mathrm{NHPm}_{3}(t)$ $N_{H P m_{1}}(t)=Q_{10}(t) \& N H P m_{0}(t)$ $N_{H P m_{2}}(t)=Q_{20}(t) \& N H P m_{0}(t)$

$$
\begin{aligned}
& +Q_{21.6}(t) \& N^{N H P m}(t) \\
& +Q_{22.5}(t) \& N_{1} \operatorname{NPm}_{2}(t) \\
& +Q_{23.4}(t) \& \operatorname{NHPm}_{3}(t)
\end{aligned}
$$


$N_{H P m}(t)=Q_{30}(t) \&\left(1+N_{H P m}(t)\right)$

Taking LST of relations (23) and solving for $\operatorname{NHPm}_{0}^{* *}(s)$. The expected numbers of hardware preventative maintenances are given by

$N_{N P m_{0}=\lim _{s \rightarrow 0} s N H P m_{0}^{* *}(s)} \operatorname{NH}_{D_{2}}$
$N_{6}=p_{02} p_{23.4} p_{30}+p_{03} p_{30}(1-$ $p_{22.5}$ )and $D_{2}$ is already mentioned.

\section{PROFIT ANALYSIS}

The profit incurred to the system model in steady state can be obtained as:

$P=K_{0} A_{0}-K_{1} B_{0}^{H}-K_{2} B_{0}^{S}-K_{3} N H R_{0}-K_{4} N S U_{0}-$ $K_{5} B_{0}^{P m}-K_{6} N H P m_{0}$

where $A_{0}, B_{0}^{H}, B_{0}^{S}, N H R_{0}, N S U_{0}, B_{0}^{P m}, N H P m_{0}$ are already defined.

\section{PARTICULAR CASES}

$\quad$ Suppose $\quad g(t)=$
and $m(t)=\gamma e^{-\gamma t}$
We can obtain the following results:

$\operatorname{MTSF}\left(T_{0}\right)=\frac{N_{1}}{D_{1}}$, Availability $\left(A_{0}\right)=\frac{N_{2}}{D_{2}}$

Busy Period due to hardware

failure $\left(B_{0}^{H}\right)=\frac{N_{3}^{H}}{D_{2}}$

Busy Period due to software

failure $\left(B_{0}^{S}\right)=\frac{N_{3}^{S}}{D_{2}}$

Busy Period due to hardware preventive

maintenance $\left(B_{0}^{P m}\right)=\frac{N_{3}^{P m}}{D_{2}}$

Expected number of repair at hardware

failure $\left(N H R_{0}\right)=\frac{N_{4}}{D_{2}}$

Expected number of up - gradation at

software failure $\left(N S U_{0}\right)=\frac{N_{5}}{D_{2}}$

Expected number of preventive maintenance

of the hardware $\left(\mathrm{NHPm}_{0}\right)=\frac{N_{6}}{D_{2}}$

Where

$$
\begin{aligned}
& N_{1}=\frac{1+b \lambda_{2}}{\left(a \lambda_{1}+b \lambda_{2}+\beta_{0}+\theta\right)} \\
& D_{1}=\frac{\left(a \lambda_{1}+b \lambda_{2}+\beta_{0}\right)\left(a \lambda_{1}+b \lambda_{2}+\beta_{0}+\theta\right)-\theta b \lambda_{2}}{\left(a \lambda_{1}+b \lambda_{2}+\beta_{0}\right)\left(a \lambda_{1}+b \lambda_{2}+\beta_{0}+\theta\right)} \\
& N_{2}= \frac{\left(a \lambda_{1}+b \lambda_{2}+\beta_{0}+\theta\right)}{\left(a \lambda_{1}+b \lambda_{2}+\beta_{0}\right)\left(a \lambda_{1}+b \lambda_{2}+\beta_{0}+\theta\right)} \\
& D_{2}= \frac{\left(a \lambda_{1}+b \lambda_{2}+\beta_{0}+\theta\right)\left(\alpha \gamma \theta\left(a \lambda_{1}+\beta_{0}+\theta\right)+\gamma \theta a \lambda_{1}\left(a \lambda_{1}+b \lambda_{2}+\beta_{0}+\theta\right)+\right.}{\theta \gamma \alpha\left(a \lambda_{1}+b \lambda_{2}+\beta_{0}\right)\left(a \lambda_{1}+b \lambda_{2}+\beta_{0}+\theta\right)\left(a \lambda_{1}+b \lambda_{2}+\beta_{0}+\theta\right)} \\
& N_{3}^{H} \frac{a \lambda_{1}\left(\left(a \lambda_{1}+\beta_{0}+\theta\right)\left(a \lambda_{1}+b \lambda_{2}+\beta_{0}\right)+a \lambda_{1} b \lambda_{2}\right)}{\alpha\left(a \lambda_{1}+b \lambda_{2}+\beta_{0}\right)\left(a \lambda_{1}+b \lambda_{2}+\beta_{0}+\theta\right)\left(a \lambda_{1}+b \lambda_{2}+\beta_{0}\right)} \\
& N_{3}^{S}= \frac{b \lambda_{2}}{\left(a \lambda_{1}+b \lambda_{2}+\beta_{0}\right)\left(a \lambda_{1}+b \lambda_{2}+\beta_{0}+\theta\right)} \\
& N_{3}^{P m}=\frac{\beta_{0}\left(a \lambda_{1}+b \lambda_{2}+\beta_{0}+\theta\right)}{\gamma\left(a \lambda_{1}+b \lambda_{2}+\beta_{0}\right)\left(a \lambda_{1}+b \lambda_{2}+\beta_{0}+\theta\right)} \\
& N_{4}=\frac{a \lambda_{1}\left(\left(a \lambda_{1}+\beta_{0}+\theta\right)\left(a \lambda_{1}+b \lambda_{2}+\beta_{0}\right)+a \lambda_{1} b \lambda_{2}\right)}{\left(a \lambda_{1}+b \lambda_{2}+\beta_{0}\right)\left(a \lambda_{1}+b \lambda_{2}+\beta_{0}+\theta\right)\left(a \lambda_{1}+b \lambda_{2}+\beta_{0}\right)} \\
& N_{5}=\frac{b \lambda_{2}}{\left(a \lambda_{1}+b \lambda_{2}+\beta_{0}\right)} \\
& N_{6}=\frac{\beta_{0}\left(a \lambda_{1}+b \lambda_{2}+\beta_{0}+\theta\right)}{\left(a \lambda_{1}+b \lambda_{2}+\beta_{0}\right)\left(a \lambda_{1}+b \lambda_{2}+\beta_{0}+\theta\right)}
\end{aligned}
$$

\section{CONCLUSION}

The reliability measures of a computer system have been obtained by assuming $g(t)=\alpha e^{-\alpha t}, f(t)=$ $\theta e^{-\theta t}$ and $m(t)=\Upsilon e^{-\Upsilon t}$. And, graphs are drawn for the particular results of these measures as shown in figures 2 to 4. It is analyzed that the mean time to system failure (MTSF), availability and profit go on decreasing with the increase of failure rates $\left(\lambda_{1}\right.$ and $\left.\lambda_{2}\right)$ and the rate $\left(\beta_{0}\right)$ by which hardware undergoes for preventive maintenance after a pre specific operation time ' $t$ ' while their values keep on moving up with the increase of hardware repair rate $(\alpha)$, software up-gradation rate $(\theta)$ and preventive maintenance rate $(\gamma)$ provided system has more chances of hardware failure. Further, it is interesting to note that a computer system has more values of these reliability measures when it has more chances of software failure than that of hardware failure. The graphical presentation of the results related to these reliability measures obtained for the system model is shown in the figures 2 to 4 . 
VII. GRAPHICAL PRESENTATION OF RELIABILITY MEASURES FOR THE SYSTEM MODEL

Fig. 2: MTSF Vs Hardware Failure Rate (ג1)

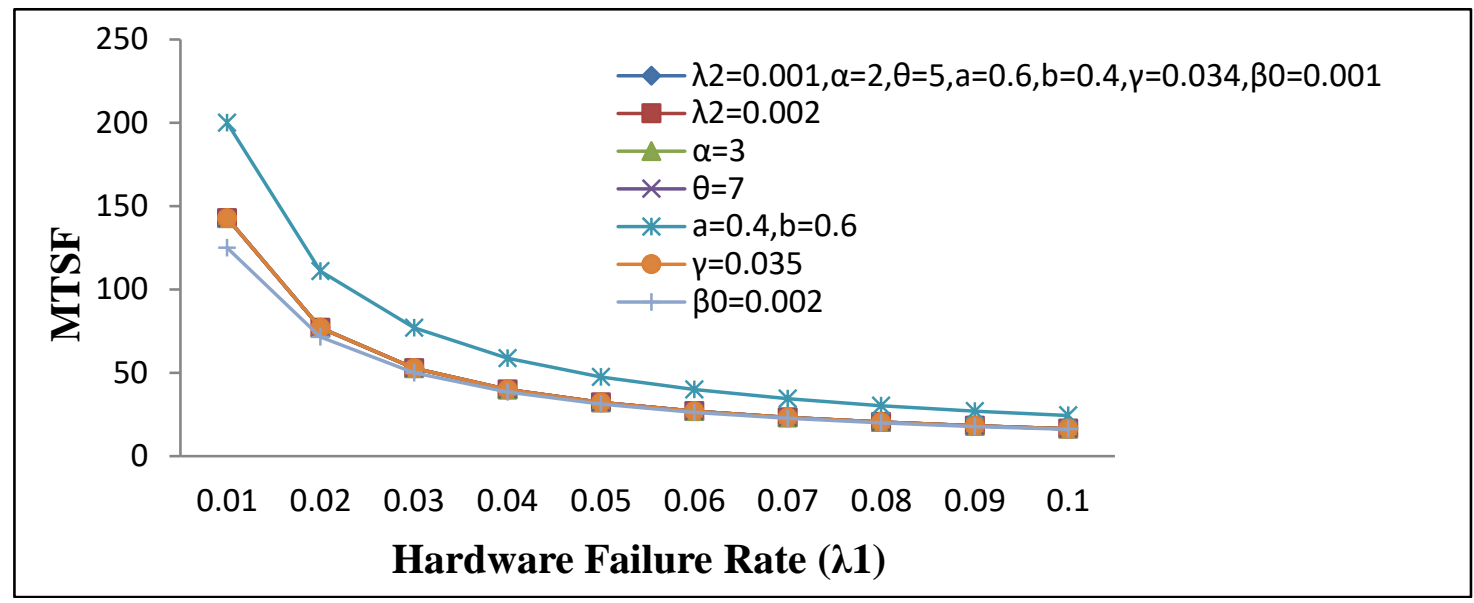

Fig. 3: Availability Vs Hardware Failure Rate ( $\lambda 1)$

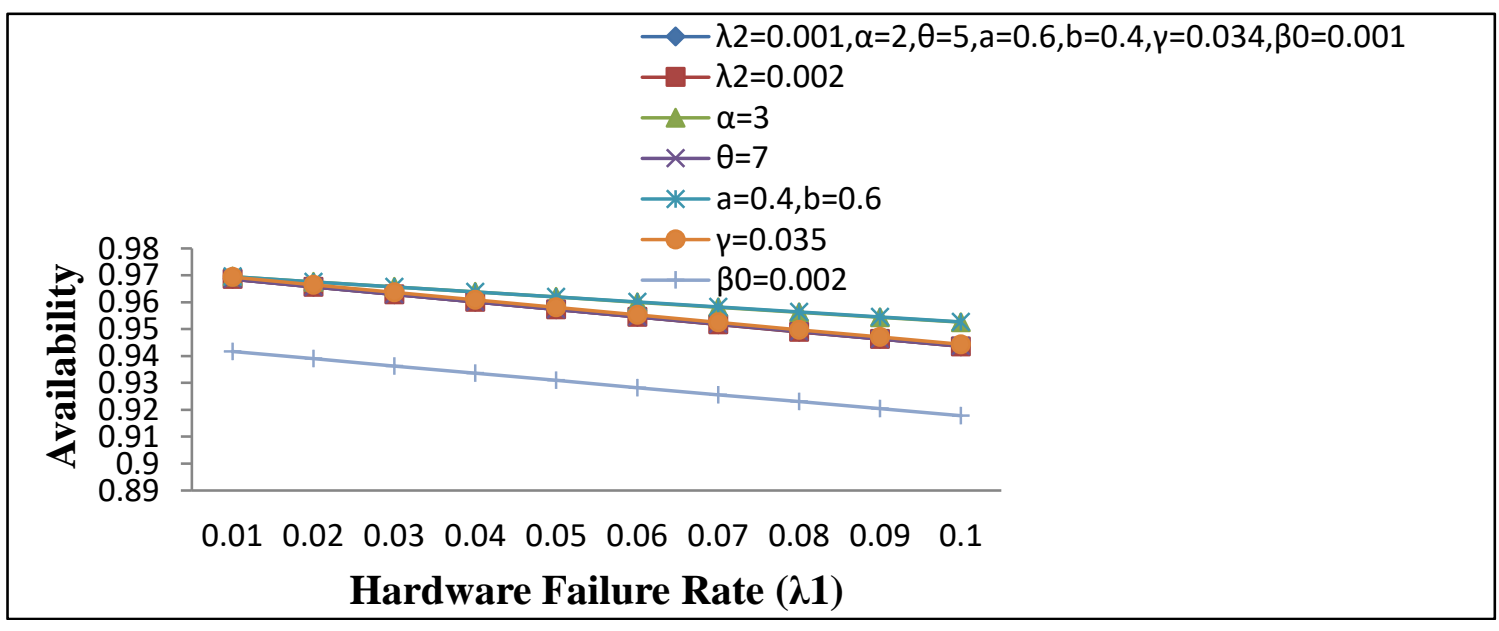

Fig. 4: Profit (P) Vs Hardware Failure Rate (ג1)

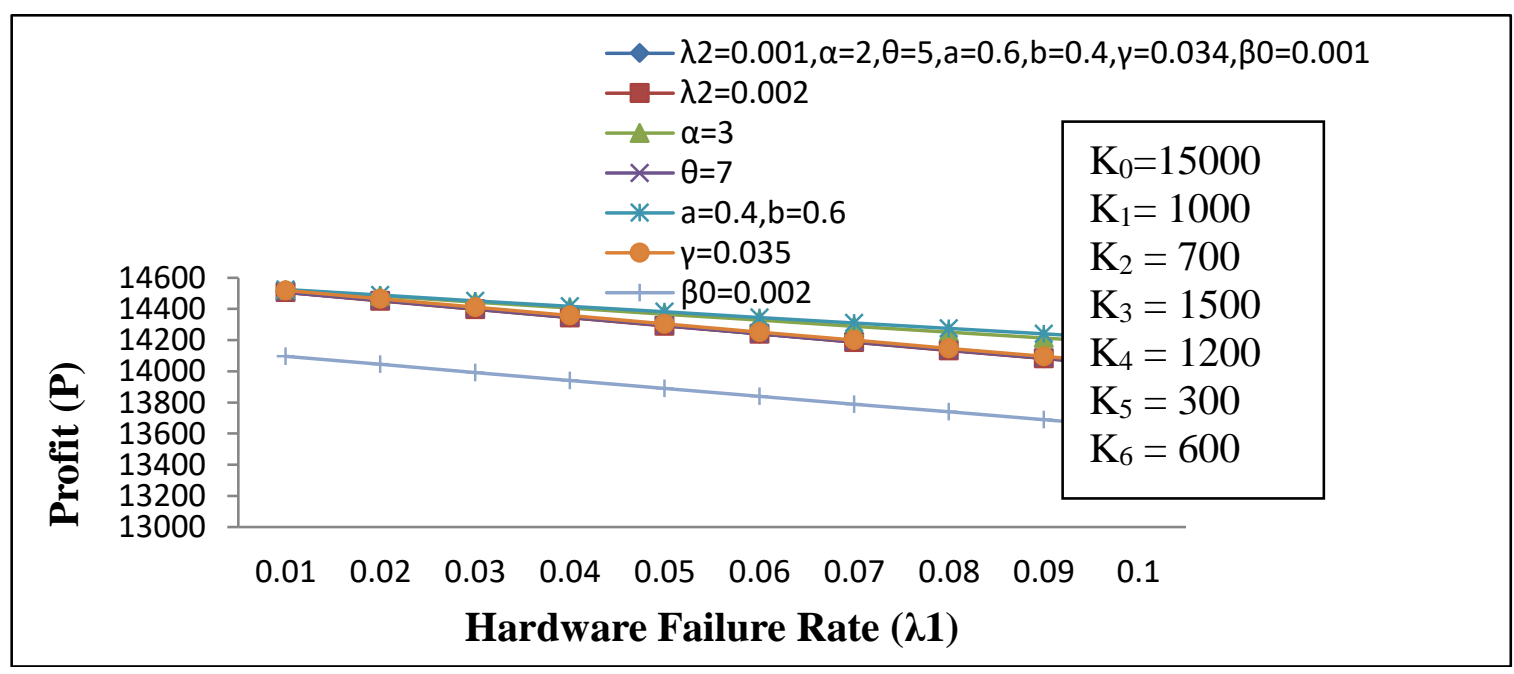




\section{COMPARATIVE STUDY OF PROFIT OF SYSTEM MODELS}

The profit of the present model has been compared with that of the model discussed in the research paper Munday and Malik (2015). It is revealed that the present model is less profitable. And, hence we can say that the concept of hardware preventive maintenance in a computer system with software redundancy in cold standby is not much helpful in making the system more profitable. The behavior of the profit difference of the system model with respect to hardware failure rate $\left(\lambda_{1}\right)$ has been shown graphically in figure 5.

\section{GRAPHICAL PRESENTATION OF PROFIT DIFFERENCES}

Fig. 5: $P-P 1$ Vs Hardware Failure Rate ( $\lambda 1)$

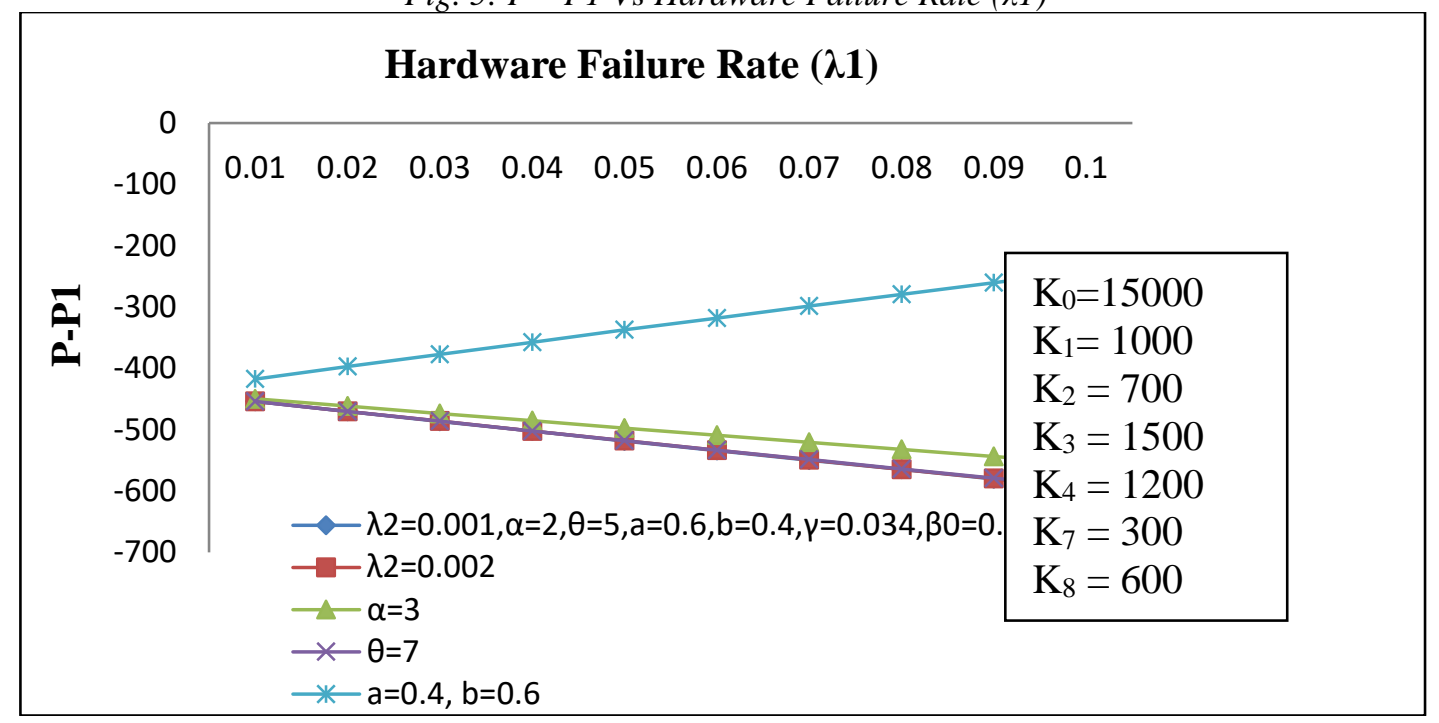

\section{REFERENCES}

[1]. S. Osaki, "Reliability analysis of a two-unit standby redundant system with preventive maintenance", IEEE Transactions on Reliability, vol. R-21 (1), pp. $24-29,1972$.

[2]. M. Rander, S. Kumar and A. Kumar, "Cost analysis of a two dissimilar cold standby system with preventive maintenance and replacement of standby", Microelectronics Reliability, vol. 34 (1), pp. $171-174,1994$.

[3]. S.C. Malik, P. Nandal and M.S. Barak, "Reliability analysis of a system under preventive maintenance", Journal of Mathematics and System Sciences, vol. 5 (1), pp. $92-115$, 2009.
[4]. S.C. Malik, "Reliability modeling of a computer system with preventive maintenance and priority subject to maximum operation and repair times", International Journal of System Assurance Engineering and Management, vol. 4 (1), pp. 94100, 2013.

[5]. V.J. Munday and S.C. Malik, "Stochastic modelling of a computer system with software redundancy", International Journal of Engineering and Management Research, vol. 5(1), pp.295-302, 2015

[6]. V.J. Munday, Permila and S.C. Malik, "Reliability modelling of a computer system with hardware redundancy subject to preventive maintenance", International Journal of Statistics and Reliability Engineering, vol. 3(2), pp. 176-189, 2016. 